\title{
Laser spectroscopic oxygen sensor using diffuse reflector based optical cell and advanced signal processing
}

\author{
J. Chen • A. Hangauer $\cdot$ R. Strzoda $\cdot$ M.-C. Amann
}

Received: 15 October 2009 / Revised version: 10 February 2010 / Published online: 25 March 2010

(C) Springer-Verlag 2010

\begin{abstract}
In this paper, a VCSEL-based oxygen sensor applied for real-time combustion optimization in furnaces is presented. A diffuse reflector instead of a spherical mirror is used to avoid alignment problems during thermal cycles the sensor is exposed to in the in-situ measurement. In spite of the signal loss, this concept proves to be suitable for measurements in the exhaust of a gas furnace even if severe condensation at the optic surfaces occurs. The optic cell has a background level in the order of $10^{-5}$ in absorbance and is long-term stable. A real-time microcontroller-suited curve fitting algorithm is applied to further improve the long-term stable operation of the whole sensor, because a drift of the spectral background is compensated. Neither absolute wavelength scale, nor absolute wavelength difference between two measurement points is needed for concentration determination. Furthermore, the gas pressure can be simultaneously determined. Kalman filtering is also applied to reduce the concentration measurement noise without losing the reliability and fast response time of the sensor. The techniques applied in the sensor are not limited to oxygen sensing, but also can be applied for sensing of other gas molecules.
\end{abstract}

\section{Introduction}

For combustion applications in gas furnaces, optimization of the burning process is very important for energy savings

J. Chen $(\bowtie) \cdot$ A. Hangauer $\cdot$ R. Strzoda

Corporate Research and Technology, Siemens AG,

Otto-Hahn-Ring 6, 80200 Munich, Germany

e-mail: jia.chen@wsi.tum.de

J. Chen · A. Hangauer $\cdot$ M.-C. Amann

Walter Schottky Institut, Technische Universität München,

Am Coulombwall 3, 85748 Garching, Germany and reduction of pollutants emission. Monitoring of the oxygen $\left(\mathrm{O}_{2}\right)$ content of the exhaust gas allows for an effective combustion control. For a large scale gas furnace with several MW power, the oxygen concentration is regulated to 1 vol\%-2 vol\% with utilization of an oxygen sensor and a control loop. Without using an oxygen sensor the burner has to be adjusted in a way that with varying gas quality and varying air density (summer/winter) the burning process is still complete, i.e. no high levels of carbon monoxide are generated. In this case the $\mathrm{O}_{2}$ content in the exhaust can be 4 vol\%-6 vol\%. For wall-hanging gas boilers in households the oxygen content is adjusted to 4 vol\%-6 vol\%, compared to 7 vol\%-8 vol\% without regulation with an oxygen sensor [1]. According to Siegert's formula [2], by reducing the $\mathrm{O}_{2}$ content in the exhaust by 4 vol\%-5 vol\% the efficiency of the furnace can be improved by approximately $1 \%$ [3] because the energy used to heat up this oxygen is no longer lost. An oxygen sensor thus enables optimum operation under different gas quality and ambient temperature conditions and eliminates the need for manual adjustment. This is especially important with a liberalized gas-market in the E.U. since the gas quality (i.e. calorific value) cannot be assumed to be constant.

The requirements for such oxygen sensors are fast time response (second range), high accuracy ( 0.1 vol\% resolution), selectivity, in-situ measurement capability, and longterm stable operation even under harsh conditions in an exhaust gas pipe. The conventional technology-the $\mathrm{ZrO}_{2}$ based lambda probes [4] are widely used in cars and also in medium or large scale heater appliances. However, these sensors suffer from several drawbacks.

- Despite the normal aging of the probe (up to three years are typically guaranteed in a furnace application), these sensors can be poisoned with heavy metals (e.g. $\mathrm{Mn}, \mathrm{Pb}$ ). 
- Grids for flame protection are needed due to the high operating temperature of the sensor, to prevent ignition of unburned gas. This again increases the response time of the sensor.

- The high operating temperature of $\mathrm{ZrO}_{2}$ sensor of above $600^{\circ} \mathrm{C}$ has to be well controlled, because any temperature variation will result in a change of the measured signal (Nernst potential).

In this paper, a tunable diode laser spectroscopy- (TDLS-) based sensor is presented as an alternative to the $\mathrm{ZrO}_{2}$ sensor for combustion optimization. While complex and rather expensive TDLS sensor systems are already employed in process monitoring applications e.g. in power plants [5], they are not yet applied in medium or small scale burners e.g. for domestic heating appliances because there compact and low cost sensors are needed.

The challenges for the laser-optic oxygen sensor are as follows.

- The sensor must be long-term stable, which means the sensor optics must withstand the temperature cycles in the exhaust gas and must not get misaligned during the whole sensor lifetime (up to 10 years). And the sensor must also be robust against condensation or contamination on the optical surfaces. These may cause a certain decrease of the light power arriving on the photodiode.

- The sensor must be compact and based on computationally efficient algorithms.

Our solution to these problems is the utilization of a diffuse reflector and a microcontroller-suited on-line curvefit algorithm, which is capable to evaluate gas concentrations in noisy signals. We employed wavelength modulation spectroscopy, with second harmonic detection [6] and leastsquares curve fitting to improve long-term stability compared to fixed detection at the absorption line center [7]. These are explained in Sects. 2 and 3, respectively. Additionally, Kalman filtering is applied to further reduce the measurement noise of the concentration value without increasing the sensor response time. Furthermore, gas pressure is estimated simultaneously using the on-line curve fit algorithm (Sect. 4).

\section{Design of the sensor optics: utilization of a diffuse reflector}

The sensor probes an $\mathrm{O}_{2}$ absorption line at a wavelength of $763 \mathrm{~nm}$, where low cost vertical-cavity surface-emitting lasers (VCSELs) are available [8]. A structural diagram of the whole sensor is shown in Fig. 1. The sensor has a reflective geometry with laser and photodetector side by side and both directed at a reflector at $10 \mathrm{~cm}$ distance. A photograph of the sensor's optic cell is shown in Fig. 2. The laser

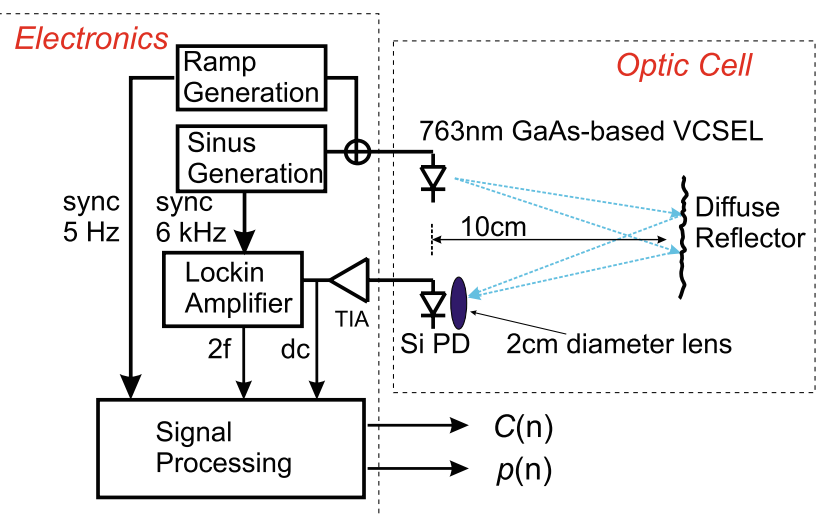

Fig. 1 Layout of the oxygen sensor consisting of an optic cell with diffuse reflective geometry and microcontroller-based electronics. $C(n)$ and $p(n)$ are the sensor outputs, which represent the $n$-th estimated gas concentration and pressure value

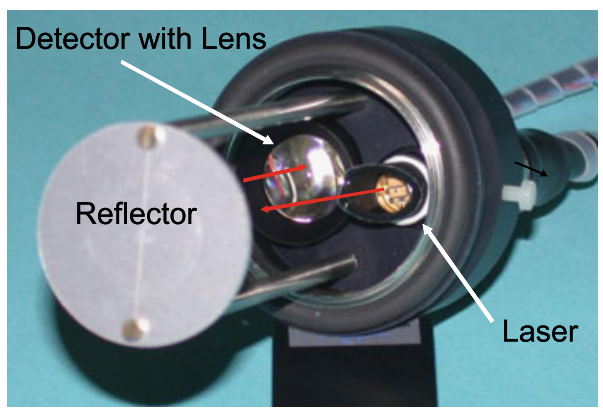

Fig. 2 Photograph of the sensor optic cell. The VCSEL emitted light is reflected by the diffuse reflector to a $2 \mathrm{~cm}$ diameter lens, which collects the light and images the speckle pattern to the $3 \mathrm{~mm}$ diameter silicon photodiode. The optical path length is $2 \times 10 \mathrm{~cm}$

beam passes through a window with Brewster angle and is scattered at the rough surface of the diffuse reflector, which is made of anodized aluminum with a surface roughness of $1-2 \mu \mathrm{m}$. The reflected light interferes at different positions on the photodetector, which results in a laser speckle pattern, i.e. a spatial distribution of interfering light modes on the surface of the detector. To collect as much light as possible, the $3 \mathrm{~mm}$ diameter silicon photodetector is equipped with a $2 \mathrm{~cm}$ diameter lens, which optically magnifies the detector area.

There are several sources of interferences in an optical cell which cause the cell background, i.e. a non-smooth transmission function of the nitrogen filled gas cell: reflections between laser and photodiode; laser speckle pattern and reflections between surfaces of optical components such as lenses or windows. In spherical mirror based cells, etalons are easily formed by the surfaces of laser and photodiode due to the high reflectivity of the mirror. These etalons have short spectral periodicity corresponding to a multiple of the physical length of the cell. For a diffuse reflector, the reflection is undirected, so that interferences with periodicities corresponding to a multiple of the cell length have a 
much lower amplitude than in a spherical mirror based cell. The second possible source for the cell background is the laser speckle pattern on the detector, which is wavelength dependent. A detailed analysis of the laser speckle pattern caused by utilization of a diffuse reflector can be found in $[9,10]$. By applying the lens, many speckle points are integrated by the photodetector, with the effect that less intensity fluctuations appear on the transmission function when the laser wavelength is tuned [11]. Furthermore, only the light reflected at close positions on the diffuse reflector can interfere on the photodiode, because the detector is placed in the imaging plane of the lens. The interfering light beams have optical path length differences in $\leq \mathrm{mm}$ range and result in fringes with long spectral periodicity. Reflections on the surface of optical components like lenses or windows of the laser and detector also cause fringes on the cell background. These components are needed for protection of the laser and detector from the combustion gas in the furnace. Their spectral period length depends strongly on the distance and thickness of these components.

The measured spectral background for the diffuse reflector-based cell is shown in Fig. 3. It has a peak to peak amplitude of $10^{-5}$. This number refers to the intensity fluctuation on $I_{2} / I_{0}$, if no gas absorption is present. $I_{0}$ is the averaged

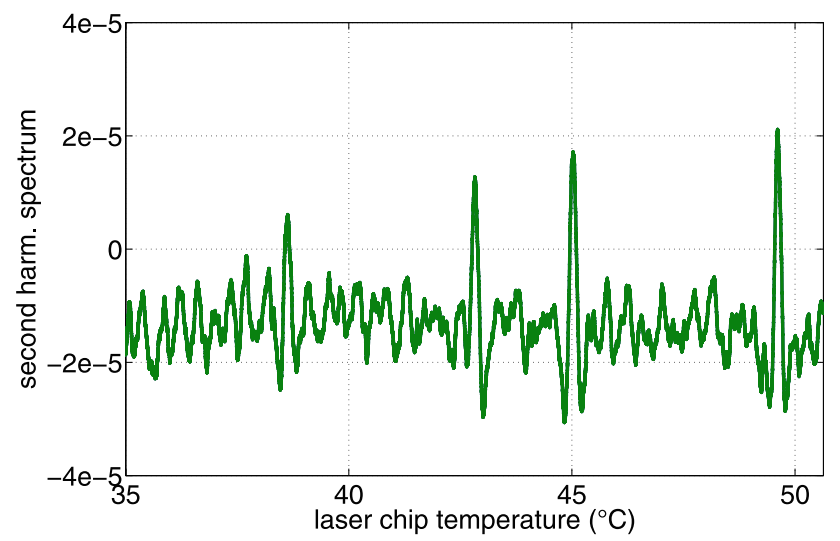

Fig. 3 The second harmonic background level of the optic cell. The high frequency etalon fringes have a peak to peak value of $10^{-5}$. Residual $\mathrm{O}_{2}$ absorption lines are present at $38^{\circ} \mathrm{C}, 43^{\circ} \mathrm{C}, 45^{\circ} \mathrm{C}, 50^{\circ} \mathrm{C}$ due to incomplete purging with $\mathrm{N}_{2}$ intensity arriving at the photodetector and $I_{2}$ is the detected power of the second harmonic. The residual interference is a structure with a dominant periodicity corresponding to the distance between laser chip and the laser window $(\sim 1.5 \mathrm{~cm})$. The detection limit is very likely to be improved if the optical cell is optimized with respect to the laser window. The baseline fringes due to diffuse reflector is probably below $10^{-5}$ and is also expected to stay stable during the time, which may not be the case for a spherical mirror based cell when a drift of adjustment occurs. The ultimate sensitivity limit is given by the photodetector noise, i.e. shot noise and dark noise, and is the order of $10^{-6}$ at $10 \mathrm{~Hz}$ bandwidth.

The most significant advantage of a diffuse reflector lies in the simplicity of alignment: almost no alignment is required and optical setup changes due to thermal cycles can be tolerated. On the contrary, focusing the light on the photodetector by a spherical mirror is very alignment-sensitive, which is difficult to maintain with thermal cycles. The gas temperature in the exhaust gas pipe during operation varies from RT to $80^{\circ} \mathrm{C}$ (condensing gas furnaces) and from RT to $120^{\circ} \mathrm{C}-170^{\circ} \mathrm{C}$ (non-condensing gas furnace) and is monitored during sensor operation.

The hot and humid combustion gas causes condensation on cold surfaces like the laser window, lens and the reflector. We found that the diffuse reflector is robust against condensation, because the light is always partly reflected to the photodetector due to many possible reflection paths. The power decreases by a factor of 5 due to condensation. In comparison, applying spherical mirror the power decreases by at least a factor of 50 due to condensation. A brief comparison between diffuse reflector and spherical mirror is shown in Table 1. The disadvantage of the diffuse reflector is the limited reflectivity, which is approximately $1 \%$ resulting in several $\mu \mathrm{W}$ photodetector power, which is nevertheless sufficient for our application.

\section{Design of the spectral evaluation algorithm-nonlinear curve fitting}

Wavelength modulation spectroscopy with second harmonic detection is employed in this sensor. The VCSEL is tuned

Table 1 Comparison between diffuse reflector cell as described in this paper and spherical mirror based cell (as in [12]) applied in the exhaust gas measurement in gas/oil furnaces

\begin{tabular}{|c|c|c|}
\hline & Diffuse reflector & Spherical mirror \\
\hline Alignment & Almost none required & $\begin{array}{l}\text { Difficult to maintain with thermal cycles, may result in total } \\
\text { vanishing of the photo current }\end{array}$ \\
\hline Power decrease due to condensation & Factor 5 & Factor 50 \\
\hline Baseline fringes due to reflector & $\leq 10^{-5}$ & $10^{-4}, \ldots, 10^{-5}$, can increase if alignment changes over time \\
\hline Reflectivity (without condensation and dirt) & $1 \% \ldots 2 \%$ & $20 \% \ldots$ approx. $100 \%$ \\
\hline
\end{tabular}


Fig. 4 Block diagram of signal processing chain for spectral evaluation

\section{Analog signals}

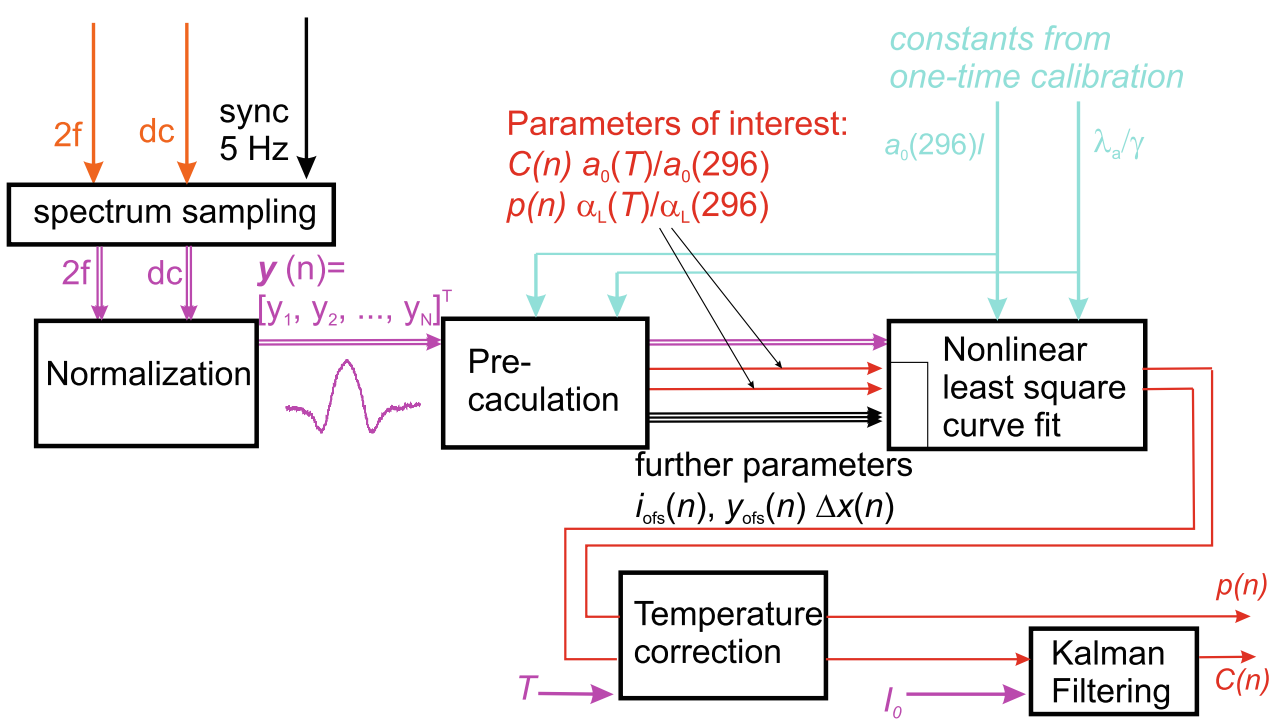

with current (Fig. 1), which is a ramp signal superimposed with a $6 \mathrm{kHz}$ sinusoidal modulation. The measurement rate ${ }^{1}$ of the one spectrum and gas concentration value is $5 \mathrm{~Hz}$. The signal processing and control are done by a compact microcontroller-based electronics (board dimensions: $10 \mathrm{~cm} \times 6 \mathrm{~cm})$.

The task of signal processing is to extract the gas concentration and pressure from the recorded gas spectra, which is described with the following steps (Fig. 4): data recording, signal normalization, pre-calculation, nonlinear leastsquares curve fit, temperature correction and Kalman filtering. After a brief introduction of signal normalization, the signal model used for curve fitting is described. Later on, the paper is focused on the pre-calculation to obtain good initial values for iterative nonlinear curve fit, whereas the curve fit algorithm itself and temperature correction to estimated concentration and pressure values are briefly discussed. Kalman filtering of the concentration values is briefly presented at last.

\subsection{Signal normalization}

The detected second harmonic power using a Lockin amplifier is dependent on the number density of absorbing molecules in the laser beam and also proportional to incident laser power which falls on the photodetector. Therefore, the second harmonic signal is normalized with the DC detector power after the transimpedance amplifier, so that the result is a pure measure of the gas absorption spectra.

If stray light falls additionally on the photodetectoreven it is unlikely in the exhaust gas pipe-the first harmonic signal at the center wavelength of the gas absorption

${ }^{1}$ The measurement rate is $1 /$ (time span between two measurements). line or the modulated DC Light (difference signal between laser turned on and laser turned off) could be used to determine the incident laser power. The normalization with first harmonic is described in (3) in [7]. But in this case the ratio between the first harmonic and laser power has to be determined in a calibration at first. This ratio depends on the laser bias current and threshold current and the laser threshold current may change when the heatsink temperature of laser changes or when the laser ages.

\subsection{Signal model for curve fitting}

The $\mathrm{O}_{2}$ A-band [13] at $763 \mathrm{~nm}$ consists of isolated lines, which do not interfere with species present in air or the exhaust gas, like $\mathrm{H}_{2} \mathrm{O}, \mathrm{CO}_{2}, \mathrm{CO}, \mathrm{NO}_{x}$ and so on [14]. The gas absorption line at ambient pressure has a Voigt profile with dominating Lorentzian halfwidth (pressure broadening) of $\sim 89 \%$ of the Voigt halfwidth, in the range of room temperature to 170 degrees (temperature range in the exhaust of a gas furnace). There is no closed analytical form expression for the Voigt profile, neither for the absorption nor for the harmonic spectra. Therefore, computation of line shape is simplified by assuming a Lorentzian-based gas absorption line profile and the error caused by neglecting the Gaussian broadening is accepted. If the absorbance is low (usually less than $10 \%$ ), the transmission for a single Lorentzian absorption line can be approximated by:

$$
\begin{aligned}
T(\lambda) & =1-\frac{C \cdot l \cdot a_{0}}{1+\left(\frac{\lambda-\lambda_{c}}{\alpha_{L}}\right)^{2}}+\left(\frac{C \cdot l \cdot a_{0}}{1+\left(\frac{\lambda-\lambda_{c}}{\alpha_{L}}\right)^{2}}\right)^{2}-\cdots \\
& \approx 1-\frac{C \cdot l \cdot a_{0}}{1+\left(\frac{\lambda-\lambda_{c}}{\alpha_{L}}\right)^{2}}
\end{aligned}
$$


with $C$ being the gas concentration in ppm, $l$ the optical path length in $\mathrm{m} . a_{0}$ is the absorption coefficient in $(\mathrm{ppm} \cdot \mathrm{m})^{-1}$ at center wavelength $\lambda_{c}$ of the gas absorption line. $\alpha_{L}$ is the HWHM of the Lorentzian-shaped gas absorption line with the same unit as $\lambda$. The maximum absorbance in this system for gas furnace application is $0.5 \%$ (20\% oxygen in $20 \mathrm{~cm}$ optical path length), the maximum relative error using this approximation is $0.25 \%$.

The recorded second harmonic spectrum $\boldsymbol{y}$ consists of discretely sampled wavelengths:

$\boldsymbol{y}=\left[y_{1}, y_{2}, \ldots, y_{N}\right]^{T}$.

It is described with the following signal model:

$$
\begin{aligned}
y_{i}= & C \frac{a_{0}(T)}{a_{0}(296)} a_{0}(296) l S_{2}\left(\left(i-i_{\mathrm{ofs}}\right) \Delta x, \frac{\lambda_{a}}{\gamma p \frac{\alpha_{L}(T)}{\alpha_{L}(296)}}\right) \\
& +y_{\mathrm{ofs}}+\epsilon_{i},
\end{aligned}
$$

with $\epsilon$ being white noise. $a_{0}$ and $\alpha_{L}$ are both temperature dependent. $a_{0}$ (296) and $\alpha_{L}(296)$ are the gas absorption coefficient and linewidth at ambient temperature $T=296 \mathrm{~K}$. $S_{2}(x, m)$ is the second harmonic component of Lorentzianshaped gas spectrum if the gas absorbance is one:

$S_{2}(x, m)=\frac{1}{\pi} \int_{-\pi}^{\pi} \frac{1}{1+(x+m \cos (z))^{2}} \cos (2 z) d z$,

with $z=2 \pi f_{m} t$. An analytical expression of $S_{2}(x, m)$ is given by Arndt [15], which is applied in the model (4). The modulation index $m=\lambda_{a} / \alpha_{L}$ is the wavelength modulation amplitude $\lambda_{a}$ normalized with $\alpha_{L}$. The variable of the $S_{2}$ function is the normalized wavelength scale $x=\frac{\lambda-\lambda_{c}}{\alpha_{L}}$.

The halfwidth $\alpha_{L}=p \gamma \frac{\alpha_{L}(T)}{\alpha_{L}(296)}$ itself is proportional to gas pressure $p$ with proportionality factor $\gamma$ and has a temperature correction term $\frac{\alpha_{L}(T)}{\alpha_{L}(296)} . p \gamma$ is equal to $\alpha_{L}(296)$. In this algorithm the temperature correction is applied after the curve fit, so that the parameter estimated in the curve fit is $p \cdot \frac{\alpha_{L}(T)}{\alpha_{L}(296)}$. By dividing the value by $\frac{\alpha_{L}(T)}{\alpha_{L}(296)}$ after curve fitting (see Sect. 3.6), the pressure $p$ is obtained. The same holds for the concentration with the curve fit parameter $C \frac{a_{0}(T)}{a_{0}(296)}$.

The model based on the Arndt formula (4) has the following five parameters:

$-x$ offset $i_{\text {ofs }}$ is the index of the line center, which is not necessarily an integer

- the distance between two spectrum sample points in normalized wavelength: $\Delta x=x_{i+1}-x_{i}$

- gas pressure without temperature correction: $p \frac{\alpha_{L}(T)}{\alpha_{L}(296)}$

- spectral baseline offset: $y_{\text {ofs }}$ (caused by laser residual amplitude modulation (RAM) [16])

- gas concentration without temperature correction: $C \frac{a_{0}(T)}{a_{0}(296)}$
The RAM present in the second harmonic signal is caused by the nonlinear current-intensity modulation behavior (PI characteristic) of the laser: the additive background on the second harmonic signal is caused by the second derivative of the PI curve. Since the cubic part of the PI curve for the utilized GaAs-based VCSEL is very small, the background is modeled with an offset only and higher order terms like a slope are omitted.

\subsection{One-time calibration}

One-time calibration with known gas concentration, pressure and temperature is done before the sensor is applied for field operation. In the calibration phase, the calibration factors for concentration $a_{0}(296) l$ and gas pressure $\lambda_{a} / \gamma$ are determined. $a_{0}$ (296) can be also obtained from the HITRAN database [14] if the absorption line to be detected is known. Usually, ambient temperature and pressure $(T=296 \mathrm{~K}$; $p=1013 \mathrm{hPa}$;) are applied in the calibration phase. The calibration can also be done during the sensor operation when the gas furnace is switched off, so the exhaust pipe is purged with fresh air with oxygen content $20.9 \%$ and ambient temperature and pressure.

\subsection{Pre-calculation: determination of the initial values for parameters}

Besides $C$ and $y_{\text {ofs }}$, the other three parameters are nonlinear to the model, i.e. $y_{i}$ changes nonlinearly with the parameters $i_{\text {ofs }}, \Delta x$ and $p$. Therefore, an iterative curve fit is needed. To enable real-time curve fitting on a microcontroller, a precalculation method to determine good initial values for the model parameters has been developed using characteristic values of the measured spectrum. The procedure is shown in Fig. 5, and explained in the following steps.

- Step 1: the maximum and the adjacent two minima of the measured data are determined. The average of the minima positions as well as the position of the maximum give the index of the line center $i_{\text {ofs }}$ (Fig. 5(a)).

- Step 2: the spectrum is averaged in a symmetrical manner around the maximum to determine $y_{\text {ofs }}$, because the second harmonic spectrum theoretically has a mean of zero (Fig. 5(b)) [17].

- Step 3: once the y offset is known, the maximum-tominimum ratio $R$ can be computed and $m$ is obtained by applying the $m(R)$ relationship numerically determined from the Arndt formula (shown in Fig. 5(c)). The initial value of gas pressure without temperature correction is determined by

$$
p \frac{\alpha_{L}(T)}{\alpha_{L}(296)}=\frac{\lambda_{a} / \gamma}{m} .
$$



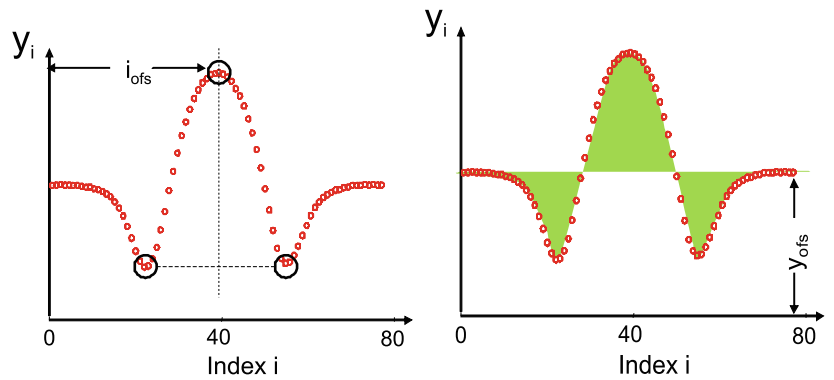

a step 1: index of maxi- $\mathbf{b}$ step 2: average of all mum point for $i_{\mathrm{ofs}} \quad$ points for $y_{\mathrm{ofs}}$

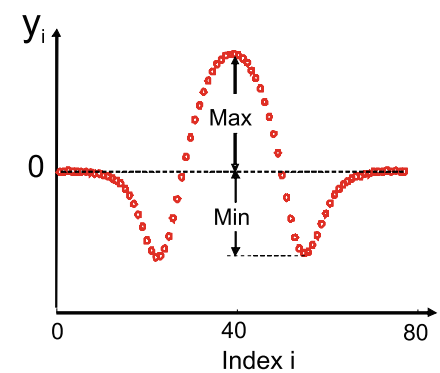

theoretical $m(R)$ behavior

$$
\text { c }
$$

step 3: $\max / \min$ ratio $(R)$ for $m$

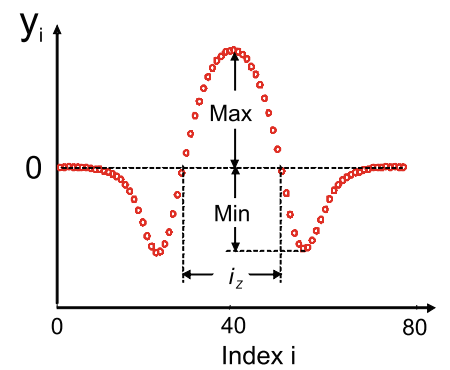

theoretical $x_{z}(R)$ behavior

d

step 4: distance of zero crossings $i_{z}$ and $R$ for $\Delta x$

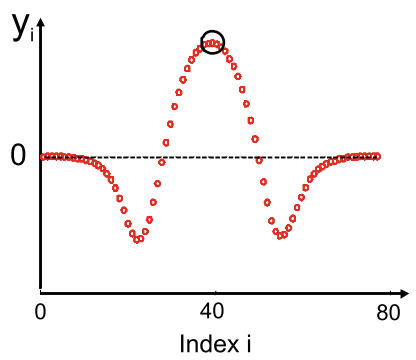

e step 5: maximum point for $C$

Fig. 5 The procedure of pre-calculation: different steps to determine the initial values for different parameters

- Step 4: the initial value of $\Delta x$ is determined by the distance between the two zero crossings. For this, the index difference $i_{z}$ and its theoretical value $x_{z}$ is divided, which gives $\Delta x=x_{z} / i_{z} \cdot x_{z}$ is given by applying the $x_{z}(R)$ relationship numerically determined from the Arndt formula. $i_{z}$ is determined from the measured spectrum (see Fig. 5(d)).

- Step 5: the initial value of $C \frac{a_{0}(T)}{a_{0}(296)}$ is determined by using maximum point of the second harmonic spectrum divided by $a_{0}(296) l S_{2}(0, m)$, while $a_{0}(296) l$ is known from the one-time calibration.

An overview of the parameters and the determination method for their initial values are shown in Table 2.

\subsection{Nonlinear least-squares curve fitting algorithms}

With these proper initial values only one to three iterations of the Levenberg-Marquardt fitting routine [18] are required to obtain a good fit to the model given in (4). This is a nonlinear least-squares curve fit, which minimizes the square error between $y$ and the signal model. The Levenberg-Marquardt algorithm requires the knowledge of the Jacobian matrix, which contains the derivatives of the model function with respect to each parameter [19]. To save computing time and capacity in the microcontroller, the Jacobian matrix is programmed analytically instead of estimating it numerically. The operating time is at least two times less compared to numerical estimation of the Jacobian matrix.

\subsection{Temperature correction}

After the nonlinear curve fit, a temperature correction according to theoretical calculations or calibration curves to the concentration and/or pressure is carried out. This is done by dividing the raw concentration and pressure values from the curve fit with the temperature correction functions $\frac{a_{0}(T)}{a_{0}(296)}$ (concentration) and $\frac{\alpha_{L}(T)}{\alpha_{L}(296)}$ (pressure).

According to HITRAN [14], we have:

$\frac{\alpha_{L}(T)}{\alpha_{L}(296)}=\left(\frac{T}{296}\right)^{\eta}$

with absorption line dependent $\eta$ in the range of $0.63-0.74$, and

$\frac{a_{0}(T)}{a_{0}(296)}=\frac{296}{T} \frac{Q(296)}{Q(T)} \frac{\exp \left(-\frac{E_{L}}{k_{B} T}\right)}{\exp \left(-\frac{E_{L}}{k_{B} 296}\right)}\left(\frac{T}{296}\right)^{\eta}$.

$Q(T)$ is the total internal partition sum for the $\mathrm{O}_{2}$ molecule [14], $E_{L}$ is the lower state energy of the transition corresponding to the absorption line and $k_{B}$ is the Boltzmann constant.

\subsection{Post signal processing-Kalman filtering}

After the curve fitting, residual noise from the sensor system is still superimposed on the concentration values. To separate the system noise from the real concentration variation, Kalman filtering is applied. Contrary to a conventional linear filter e.g. Wiener filter [20], the Kalman filter [21] is time-variant. It is a least-squares estimator in the context of time-variant models. Its "bandwidth" changes over time depending on the actual situation of the sensor and gas, e.g. 
Table 2 The linear/nonlinear parameters of the model as well as the methods to determine their initial values. The two parameters $C \frac{a_{0}(T)}{a_{0}(296)}$ and $p \frac{\alpha_{L}(T)}{\alpha_{L}(296)}$ are the parameters of interest

\begin{tabular}{lll}
\hline Model Parameter & Definition & $\begin{array}{l}\text { Spectrum characteristic values applied } \\
\text { for initial value determination }\end{array}$ \\
\hline$i_{\mathrm{ofs}}$ & $x$ offset (unit: index) & The position of max/mins \\
$y_{\mathrm{ofs}}$ & $y$ offset (unit: absorbance) & Mean value of the whole spectrum \\
$p \frac{\alpha_{L}(T)}{\alpha_{L}(296)}$ & Gas pressure without temperature correction (unit: bar) & Max to min ratio $R$ \\
$\Delta x$ & Normalized wavelength scale & $R$ and distance between zero crossings $i_{z}$ \\
$C \frac{a_{0}(T)}{a_{0}(296)}$ & Gas concentration without temperature correction (unit: $\mathrm{ppm})$ & Maximum value \\
\hline
\end{tabular}

the variance of system noise introduced by the sensor itself and real gas concentration fluctuation. In this paper, the signal model given by Riris [22] is applied and it is assumed that real concentration fluctuations and the sensor system noise are two independent processes, i.e. their variations are independent. The variance of the sensor system noise is determined theoretically from the received DC detector power $I_{0}$. By subtracting it from the total variance of the estimated concentration, the variance of real concentration fluctuation is obtained. Details of this concept is planned to be outlined in a separate letter.

\section{Sensor performance: concentration and pressure measurement}

The sensor was mounted in the exhaust pipe of a wallhanging condensing gas boiler with $10-20 \mathrm{~kW}$ heat power. The oxygen concentration was measured when the gas furnace was switched on and off for several times which is comparable to real operation. During on switching, the photodetector current decreases by a factor of $\sim 3$ due to condensation (Fig. 6). Contamination on optic surfaces turned out to be no issue in a gas furnace because of the high purity of the natural gas used for combustion. Reference measurements with electrochemical cell and lambda probe are carried out, compared to which, TDLS-based sensor shows a faster response time [23]. The real-time least-squares curve fit algorithm based on the advanced pre-calculation is implemented on a microcontroller with $24 \mathrm{MHz}$ clock rate. The fitting result is shown in Fig. 7. The received detector power in this case is $500 \mathrm{nW}$ and the detected power of second harmonic component is $500 \mathrm{pW}$, which is even two times lower than the lowest measured intensity with a wallhanging gas boiler. The fit guarantees long-term stability of the sensor.

The standard deviation of the measurement i.e. the measurement uncertainty during boiler operation for one constant concentration value is $930 \mathrm{ppm}(1 \sigma$ in $5 \mathrm{~Hz})$ without Kalman filter. For $1 \mathrm{~Hz}$ measurement, this value is converted

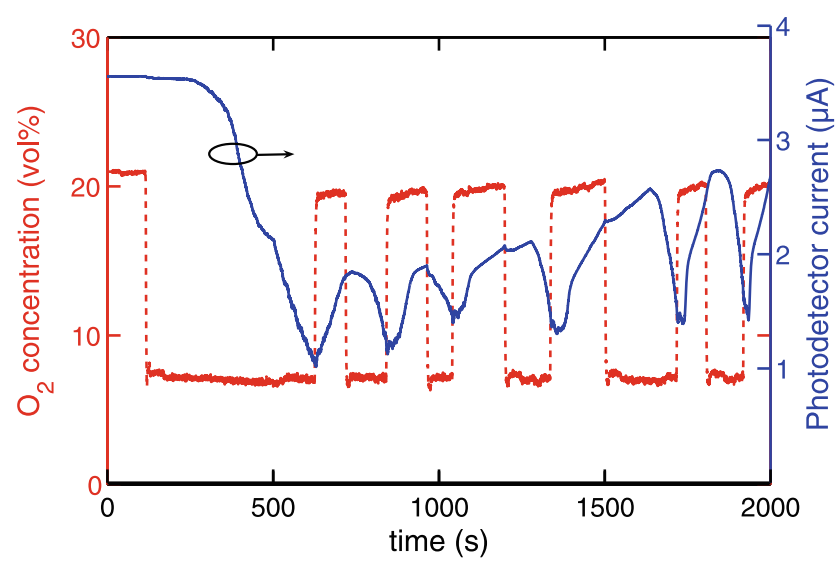

Fig. 6 The estimated concentration value during burner on/off switching. The photodetector current decreases by a factor of 3 due to condensation in this measurement. A decrease of factor 5 is observed on a longer time scale

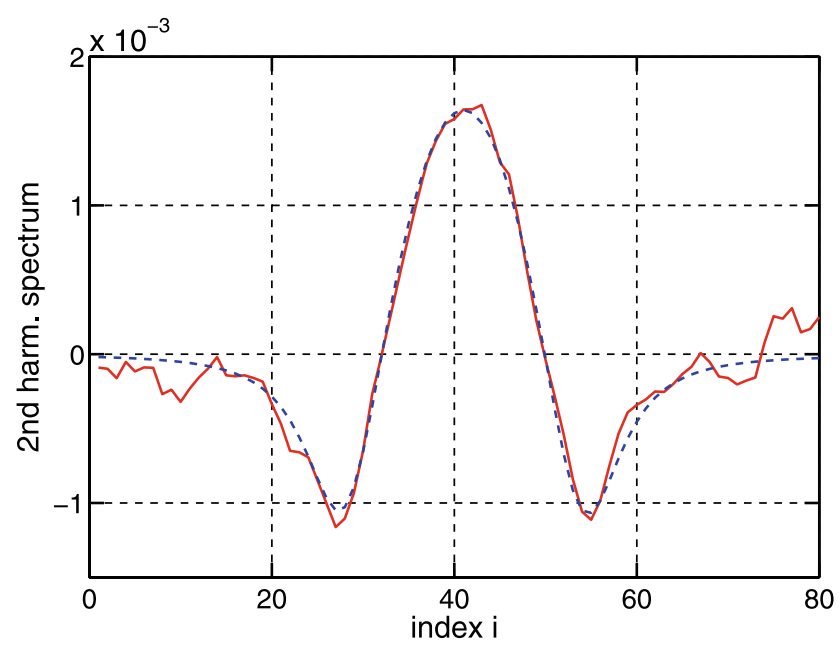

Fig. 7 Measured second harmonic spectrum (solid curve) and its minimal mean square curve fit with the model (4) (dashed curve). The detected photodetector power is $500 \mathrm{nW}$ and the power of detected second harmonic is circa $500 \mathrm{pW}$

to 417 ppm by dividing by $\sqrt{5}$, which corresponds to an absorbance of $1.1 \times 10^{-5}$. With the utilization of Kalman filter, 250 ppm standard deviation with $5 \mathrm{~Hz}$ is achieved (Fig. 8). 


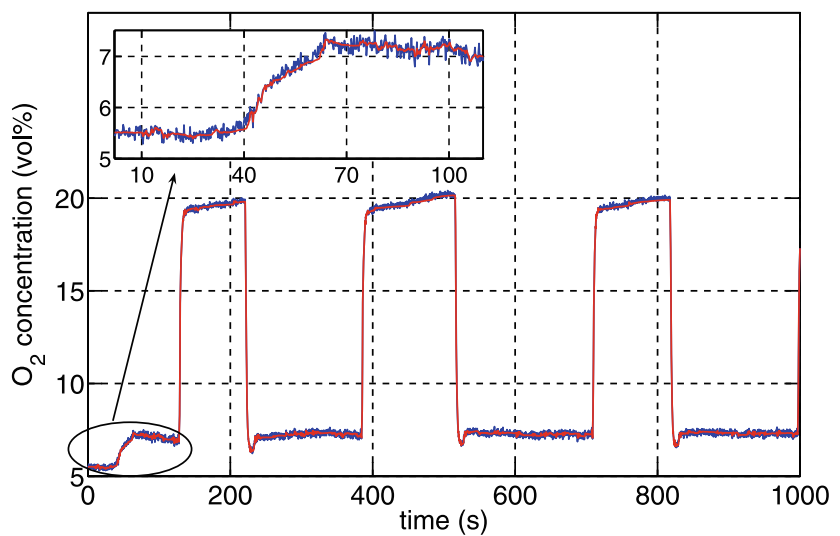

Fig. 8 The measured $\mathrm{O}_{2}$ concentration (blue) and filtered result with Kalman filter (red). Unlike conventional lowpass filter, no detection delay is introduced additionally by the Kalman filter compared to the unfiltered sensor data

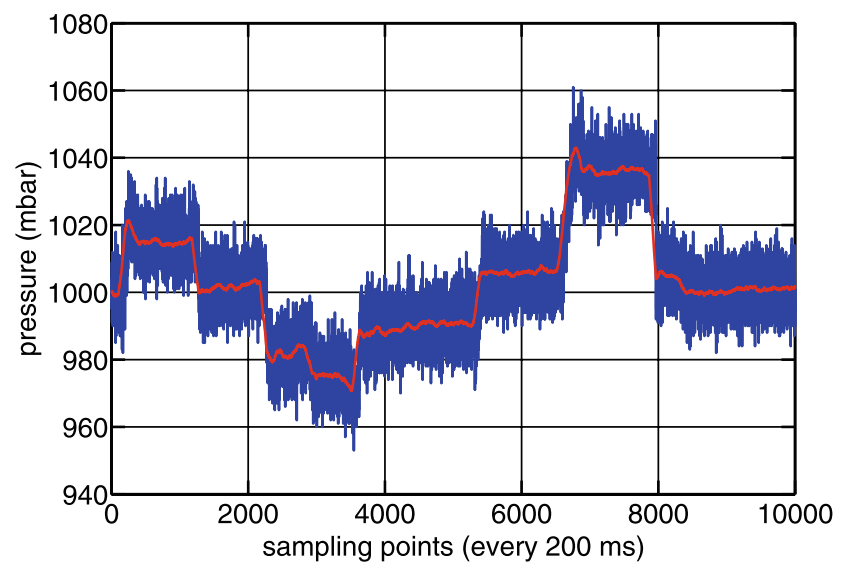

Fig. 9 Pressure measurement in every $200 \mathrm{~ms}$ (blue) and the $20 \mathrm{~s}$ average $(r e d)$. The pressure values were set manually to 1000, 1030, 1000, 970, 962, 973, 1000, 1049 and 1000 mbar. The measurement is done with $100 \%$ oxygen and at ambient room temperature

No additional detection delay is introduced by the Kalman filter compared to the unfiltered sensor data, which is very important for measurement in furnace, where fast control loops are needed.

We also show the pressure measurement in Fig. 9, wherein a nonlinear response of the sensor to pressure values is observed. The deviation is lead back to the following reasons: the assumption of a Lorentzian-based absorption line profile is probably too approximate for a pressure determination with high precision; furthermore, the laser RAM influence [24] on the spectrum is only considered as an offset in the curve fit. Nevertheless, the pressure output values are reproducible, so a nonlinear correction can be done if gas pressure is to be estimated. The standard deviation of the estimated pressure measurement is $6 \mathrm{mbar}$ at $5 \mathrm{~Hz}$ measurement rate. After nonlinear correction, the standard deviation would be approximately 9 mbar.

\section{Conclusion and discussion}

An oxygen sensor for combustion optimization in gas furnaces was developed, the measurement was realized in the exhaust gas pipe of gas furnace. Utilizing a diffuse reflector, the problem of possible misalignment of the optics due to thermal cycles, the condensation problem on optic surfaces and the problem of etalon fringes are solved. A real-time least-squares curve fit algorithm is presented, which improves the long-term stability of the sensor compared to fixed detection at the absorption line center, because the baseline drift is compensated. Real-time operation with a microcontroller is assured by determining good initial values using characteristic values of the second harmonic spectra (less iterations) and by programming the Jacobian matrix analytically (less computing complexity). There the maximum-to-minimum ratio $R$ of the second harmonic curve is a very important characteristic value, it fully represents the curve shape, whereas the gas concentration is only a $y$-scale factor of the curve once the curve shape is fixed. Neither absolute wavelength scale, nor absolute wavelength difference between two measurement points is needed for concentration determination. Kalman filtering is applied to improve the detection sensitivity without losing the reliability and the response time of the sensor operation. Both concentration and pressure measurements are carried out in the gas furnace and the detection limit of concentration is $250 \mathrm{ppm}$ in $5 \mathrm{~Hz}$. Further application such as flame-on monitoring, which is an important safety issue in burners, is possible with TDLS-based $\mathrm{O}_{2}$ sensors because of their fast response time and intrinsic self-checking capability in contrast to $\mathrm{ZrO}_{2}$-based sensors [4]. The diffuse reflector based optical cell and described signal processing are not limited to oxygen sensing, but also can be applied for sensing of other gas molecules.

Acknowledgements The authors gratefully acknowledge the financial support by the Federal Ministry of Education and Research of Germany (Project 'NOSE', No. 13N877). The first author also would like to thank Dr. Ralf Meyer for careful proofreading and Armin Spitznas and Hans Link from Siemens Building Technology for technical consultation on combustion in furnaces.

\section{References}

1. A. Spitznas, H. Link, Siemens building technologies. Private communication (2008)

2. National Industrial Fuel Efficiency Service LTD, The Boiler Operators Handbook (Graham \& Trotman Limited, 1989)

3. Buderus Heiztechnik GmbH (ed.), Handbuch für Heizungstechnik (Beuth Verlag GmbH, 2002)

4. E. Logothetis, in Proceedings of the 8th Automotive Materials Conference: Ceramic Engineering and Science Proceedings, vol. 1 (1980), p. 281

5. H. Teichert, T. Fernholz, V. Ebert, Appl. Opt. 42, 2043 (2003)

6. J. Reid, D. Labrie, Appl. Phys. B: Lasers Opt. 26, 203 (1981) 
7. T. Iseki, H. Tai, K. Kimura, Meas. Sci. Technol. 11, 594 (2000)

8. M. Grabherr, D. Wiedenmann, R. Jaeger, R. King, Proc. SPIE 5737, 120 (2005)

9. D. Masiyano, J. Hodgkinson, R. Tatam, Appl. Phys. B: Lasers Opt. 90, 279 (2008)

10. D. Masiyano, J. Hodgkinson, R.P. Tatam, Appl. Phys. B 100 (2010) This Issue. DOI:10.1007/s00340-010-4020-z. Special Issue of 7th Tunable Diode Laser Spectroscopy Conference

11. J.W. Goodman, Laser Speckle and Related Phenomena (Springer, Berlin, 1975), Chap. 2

12. J. Chen, A. Hangauer, R. Strzoda, M. Fleischer, M.C. Amann, Proc. Chem. 1(1), 1383 (2009). Proceedings of the Eurosensors XXIII Conference

13. K.J. Ritter, T.D. Wilkerson, J. Mol. Spectrosc. 121, 1 (1987)

14. L.S. Rothman, et al., J. Quant. Spectrosc. Radiat. Transf. 96, 139 (2005)

15. R. Arndt, J. Appl. Phys. 36, 2522 (1965)
16. P. Kluczynski, Å.M. Lindberg, O. Axner, Appl. Opt. 40, 770 (2001)

17. A. Hangauer, J. Chen, M.-C. Amann, Appl. Phys. B 90, 249 (2008)

18. D.W. Marquardt, J. Soc. Ind. Appl. Math. 11, 431 (1963)

19. A. Bjorck, Numerical Methods for Least Squares Problems (SIAM, Philadelphia, 1996)

20. N. Wiener, Extrapolation, Interpolation, and Smoothing of Stationary Time Series (Wiley, New York, 1949)

21. R.E. Kalman, Trans. ASME—J. Basic Eng. 35 (1960)

22. H. Riris, C.B. Carlisle, R.E. Warren, Appl. Opt. 33, 5506 (1994)

23. A. Hangauer, A. Spitznas, J. Chen, R. Strzoda, H. Link, M. Fleischer, in Procedia Chemistry, vol. 1 (2009), p. 955. Proceedings of the Eurosensors XXIII conference

24. P. Kluczynski, J. Gustafsson, Å.M. Lindberg, O. Axner, Spectrochim. Acta Part B: At. Spectrosc. 56, 1277 (2001) 